\title{
Study of the Flow Structure of A Thermal Plume Evolving in an Unlimited and in A Semi-Enclosed Environment
}

\author{
${ }^{1}$ Jamil Zinoubi, ${ }^{2}$ A. Ould Mohamed Mahmoud, ${ }^{3}$ Taoufik Naffouti, ${ }^{3}$ Rejeb Ben Maad, and ${ }^{3}$ Ali Belghith \\ ${ }^{1}$ Preparatory Institute of the Engineers Studies of Nabeul, Academic Campus \\ El Merazka, 8000 Nabeul, Tunisia \\ ${ }^{2}$ Faculty of Sciences and Techniques of Nouakchott, Department of Physics, BP 5026 \\ Nouakchott, Mauritania \\ ${ }^{3}$ Faculty of Sciences of Tunis, Department of Physics \\ Laboratory of Energizing and Thermal and Mass Transfers, El Manar 2092, Tunis, Tunisia
}

\begin{abstract}
The basic objective of this study was to better knowledge the fine flow structure of thermal plume created by a heated disk, evolving in an unlimited and in a semi-enclosed environment. The flow visualization and the study of the temperature fluctuations spectrum show clearly a considerable change of the turbulent structure of the internal flow in comparison with the free plume. This difference is especially caused by the thermosiphon effect. Indeed, this study shows that the thermosiphon affects the flow structure plume and causes the appearance of a supplementary zone, just at the system entrance, that is added to the two classic zones concerning the free plume, mentioned in the previous works. This zone is characterised by two symmetrical rotating rolls created in the vicinity to the hot source. The energy spectrum study permits to obtain certain spectral power laws, which characterize the energy transfer between the structures that are present in the flow. In fact, three qualitatively different regions were identified: first, a production region, secondly, a region with behavior as per $\mathrm{n}^{-3}$ associated with a buoyancy region and; finally, a dissipation region associated with an $\mathrm{n}^{-7}$ law. The $\mathrm{n}^{-1}$ Tchen low is observed for the free plume in the large frequency region. The existence of the $\mathrm{n}^{-1}$ region is related to an important turbulent production. These spectral regions characterize the energy transfers mechanisms among the length scales of flow investigated here.
\end{abstract}

Key words: Turbulent natural convection; thermal plume; thermosiphon flow; visualization; spectral density; cascade concept of Kolmogorov

\section{INTRODUCTION}

An important class of natural convection flows is the one, which relates to buoyancy driven flows moving freely, without the constraining influence of a surface. Such flows arise very frequently in the nature, due to thermal gradients caused by natural processes, such as those due to solar heating, evaporation and condensation, and are of considerable interest in meteorological and atmospheric studies. In technology, thermal energy and chemical waste products are rejected to the atmosphere. The plume coming out of a chimney or a cooling tower and the effluent discharged from the condensers of a power plant.

Most previous investigators have focused their attentions on the structure of the turbulent buoyant free plume (in external flows driven primarily by buoyancy forces). This type of flow has been a subject of several theoretical and experimental investigations ${ }^{[1-6]}$.

This situation (a fluid of infinite extent) is a difficult one to obtain and the fluid is generally of finite extent (internal flow), enclosed by surfaces, which may significantly affect the flow. It is, therefore, important to determine the effect of the neighbouring surfaces on the flow development mechanisms. The effect would relate to both the transient and the steady phenomena. There are also several applications in which natural convection occurs in enclosures. Some of these, such as flow in heated tubes and channels, the flows arising in rooms due to heat sources, in fluid layers enclosed between parallel plates, and in many manufacturing systems are all internal natural convection problems.

The flow structure becomes much more complicated for the interaction between a free plume and its physical surrounding arises very frequently in practice. This interaction generates a very complex mechanism $^{[7-10]}$. The plume in enclosures is not independent of the flow at the walls (Thermosiphon flow). The two flows are obviously coupled in most cases. This coupling introduces a considerable complexity in the analysis of internal flows. The problem of interaction was the subject of only few studies, among which one can mention: the effect of an adjacent vertical surface on plane plume flow ${ }^{[11,12]}$, the interaction between two thermal plumes of equal and also unequal heat ${ }^{[11-15]}$.

Corresponding Author: Jamil Zinoubi, Preparatory Institute of the Engineers Studies of Nabeul, Academic Campus, El Merazka, 8000 Nabeul, Tunisia, Tel.+ 21698213 316; Fax.+ 21671560723 
In this work, an experimental investigation of a plume, generated from a heated disk, evolving in quiet environment is described. This plume is subjected to an interaction with the wall of a vertical cylinder surrounding it. This study is focussed on understanding the mechanisms of development of this buoyancy drive flow.

\section{Experimental apparatus and measurement} techniques: The experimental apparatus is shown in Fig.1. The numbers in the description presented below refer to the part numbers of Fig.1.The thermal plume is created by a flat disk ${ }^{(2)}$ having a $0.07 \mathrm{~m}$ diameter. The plume source is electrically heated at $300^{\circ} \mathrm{C}$ on the surface by Joule effect. The disk is placed inside the cylinder, at $5 \mathrm{~cm}$ of the entrance of the open ended steel vertical cylinder ${ }^{(3)}$, which has a $0.15 \mathrm{~m}$ diameter and a $0.5 \mathrm{~m}$ height. The system is placed on a frame ${ }^{(1)}$ at 80 $\mathrm{cm}$ above the ground to allow air supply from below. The uniformity of the source surface temperature is obtained by the use of wire resistors mounted behind the disk. A thermal regulation apparatus kept the temperature of the disk as uniform as possible within a good approximation. Al-Cr thermocouples are used to measure the surface temperature of the disk. The difference in temperature between the end and the centre of the disk is less than $5^{\circ} \mathrm{C}$. The vertical cylinder was thermally insulated by an Armaflex cylindrical bed, which has a $0.02 \mathrm{~m}$ thickness ${ }^{(4)}$.

The strong dependence of the flow to the surrounding conditions, require making evolve him inside a quiet atmosphere; so, the experimental device has been placed in an independent closed room.

To explore the voltage signals corresponding to the temperature we have used the resistant wire anemometer ${ }^{(5)}$ at constant current ${ }^{(6)}$. This technique adopted for a long time by Doan Kim-Son et al. ${ }^{[16]}$, in natural convection, is based on the principle of the resistance variation of a platinum wire $(7.5 \mu \mathrm{m}$ in diameter).

In order to avoid the disruption of the flow, the probe is introduced vertically, by the system exit, so that its sensible wire was perpendicular to the ascending flow. The wire thermal inertia, where its time constant is the order of $1 \mathrm{~ms}$, introduced not of measure mistake, especially to the weak frequencies met in plumes ${ }^{[11,14]}$. Mistakes coming from the probe calibration are lower than $1 \%$.

On the other hand, in order to explore thermal voltage signals at every level of the flow, we have used a displacement system ${ }^{(7)}$ of the probe ${ }^{(5)}$, piloted by a computer $^{(8)}$, following two directions. The minimal displacement in the vertical direction is $10^{-3} \mathrm{~m}$, whereas in the horizontal direction is $2.10^{-5} \mathrm{~m}$.

A computer ${ }^{(8)}$ provided of a data acquisition card permits to appropriate of the instantaneous measures all $10 \mathrm{~ms}$ and to record signals for further statistic processing.

The visualization system used, during this study, was constituted of a He-Ne laser ${ }^{(9)}$ with a power of
$35 \mathrm{~mW}$, an electric vibrant plate ${ }^{(10)}$, a digital camera ${ }^{(11)}$ and a smoke distributor.

On the vibrant plate is glued a plane mirror of good quality, that receives a horizontal laser beam and it reflects according to the cylinder vertical axis; the displacement of the plate makes revolve the mirror around a horizontal axis, creating a plane laser sheet ${ }^{(12)}$ on the entire transverse section of the cylinder. By natural aspiration, distributor allows smoke to impregnate all the flow without any disruption.

\section{RESULTS AND DISCUSSION}

Flow visualization: The flow visualization performed by illuminating the flow by a plane laser sheet is presented in Fig. 2-8. These instantaneous photographs show the behavior of the flow in the two studied cases (a fluid of infinite and finite extent). For the Fig. 3 and 4 , the sowing of the flow is oriented toward the region close to the hot source border. On the other hand, for the other figures, the sowing takes place all along the diameter of the cylinder. Indeed, for the free plume case (plume evolving in an unlimited environment), the Fig. 2 shows the entrainment of fresh air from the surroundings. It also shows that the plume flow is constituted of the vortex of large size evolving mainly in the vertical direction. In the zone close to the source, this photo shows that the fluid coming from the low attracted by the hot surface before being driven by the ascending flow of the plume.

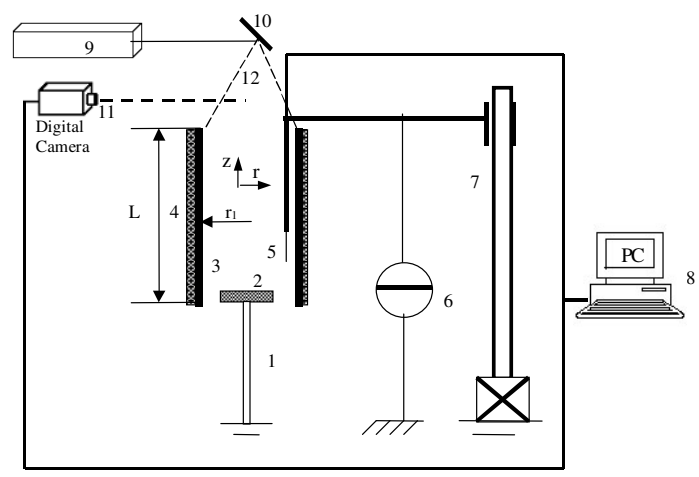

Fig. 1: Experimental configuration

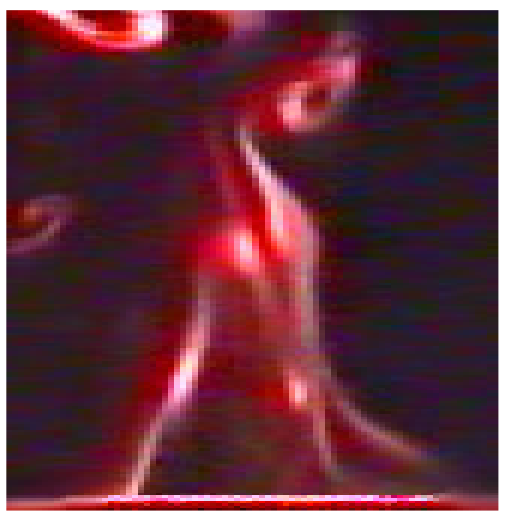

Fig. 2: Free plume 


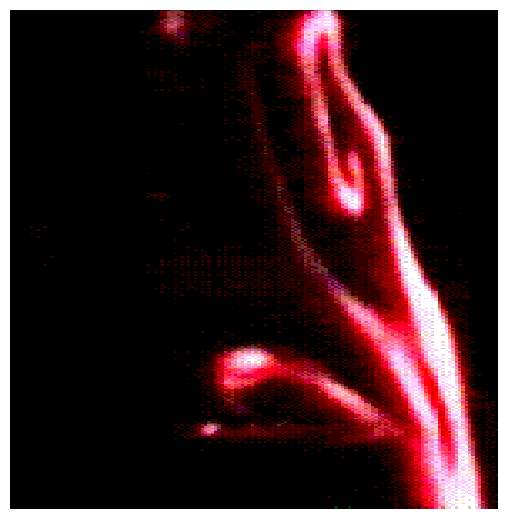

Fig. 3: The three fluid threads

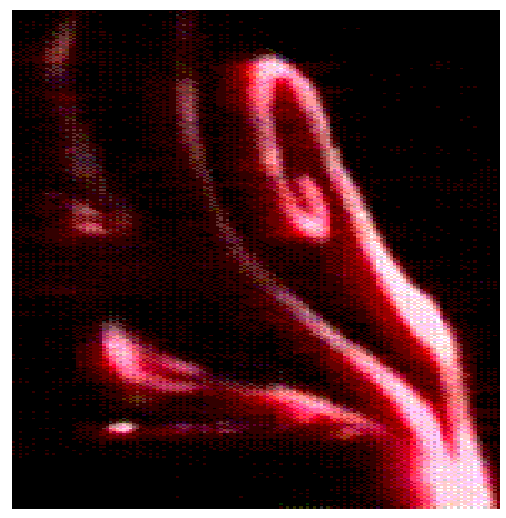

Fig. 4: The three fluid threads

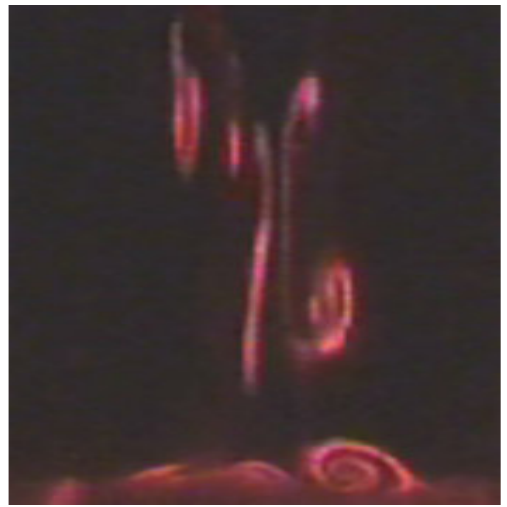

Fig. 5: Formation of the rotating rolls

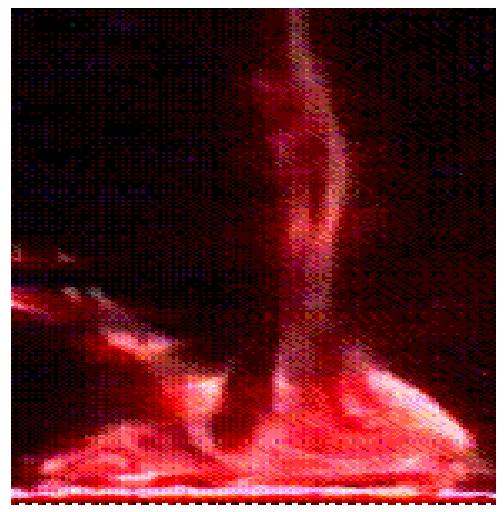

Fig. 6: Exhausts of the left roll

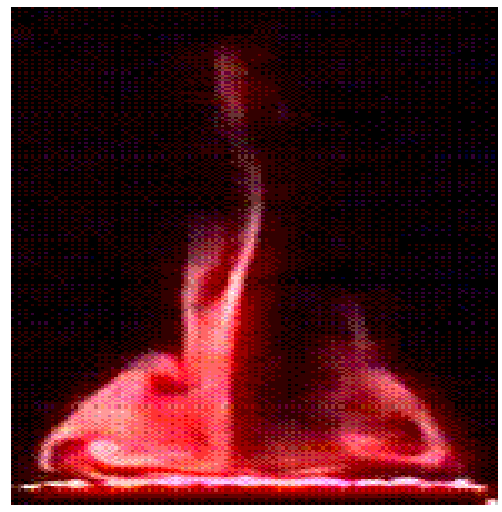

Fig. 7: Exhausts of the right roll

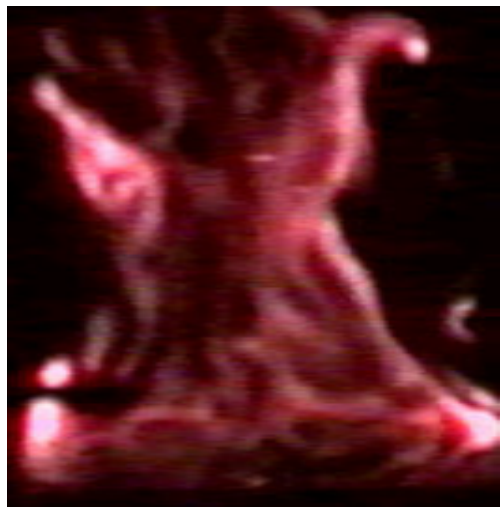

Fig. 8: Contraction and expansion of the plume

For the plume-thermosiphon case (Plume evolving in a semi-enclosed environment), the Fig. 3-8 show that the plume and the cylinder wall supply themselves regularly in fresh air by the low. The fresh air arriving from the outside, by every side of the hot source, divides in three fluid threads (Fig. 3 and 4). The first, attracted by the hot source, was directed to the disk center. The second, relatively close to the cylinder wall, sudden a strong entrainment by the plume before coming back to feed the flow along the cylinder wall. The fluid threads of the middle, it is submitted to an interaction between the plume and the cylinder wall, before pursuing its path directly toward the central part of the flow.

In the vicinity of the hot source the instantaneous photos showed, above the hot disk, the development of two rotating rolls symmetrical to the plume axis (Fig. 5). The formation of these rolls could be explained by the presence of trapped hot air near the source. In fact, the first fresh air thread, arriving from the outside, find them on the top of the relatively hotter and less dense fluid layers adjacent to the hot surface. This is an unstable fluid stratification that results in a convective motion leading to the formation of a vortex that is created close to the hot source. The trapped fluid continues to turn. When this air mass acquires a sufficient energy, it escapes vertically (Fig. 6 and 7). While escaping, it carries the first air thread and directed toward the plume axis. To replace this fluid, 
this region is supply again in fresh air that will undergo, the same process. We note the existence of a certain alternation however between the formation and the exhaust of these two rolls. Indeed, these two mechanisms don't occur simultaneously; as one of the rollers escapes, the other forms.

On the other hand, these photos show that the supply fresh air of the flow along the cylinder wall is influenced by the formation-exhaust cycle of the rotating rolls. Indeed, as one of the rollers forms, this thread is pulled completely of the wall (Fig. 4). Thereafter, it is repulsed toward the cylinder wall, where it will be blocked and driven by a downward flow along the walls. This blockage results from the lateral expansion of the plume in the superior part of the flow.

As for the fluid thread of the middle, it constitutes a provision source, in fresh air, for the flow. By its intermediate position between the two mentioned previously fluid threads, this one undergoes an interaction more or less strong with the plume and the cylinder wall. In fact, the influence that the hot source exercises on this fluid thread is also bound, as previously, to these same mechanisms of formation and exhaust of the rolls.

Otherwise, we notice that the ascending flow undergoes a contraction from a height adjoining the $5 \mathrm{~cm}$ of the source. Thereafter, it enlarges to reach the walls to a height close to $15 \mathrm{~cm}$ where vortexes, of different sizes, occupy the entire section of the cylinder (Fig. 8).

The visualization indicates the existence of three different structure zones of the flow vertical development. Therefore, in the first zone in the vicinity of the hot source $(z \leq 5 \mathrm{~cm})$, This visualization shows, above the hot disk, the development of two rotating rolls symmetrical to the plume axis. In the intermediary zone $(5 \mathrm{~cm}<z \leq 15 \mathrm{~cm})$, we observe a contraction of the plume and an ascending strong axial flow caused by buoyancy forces. In the third zone $(\mathrm{z}>15 \mathrm{~cm})$, this visualization shows the appearance of new flow behaviour where a small structure occupies all the upper part of the cylinder. The existence of these turbulent dissipating structures leads to a uniform flow in the cylinder exit.

Spectral density of the temperature fluctuations: To better understand the flow turbulent structure, a spectral analysis using Fast Fourier Transformation (FFT) was carried out. The temperature spectral density $\mathrm{E}_{\mathrm{t}}(\mathrm{n})$ obtained at the entrance and the top of the cylinder for different positions of the probe investigated at the same level, is standardised under the following from:

$$
\int_{0}^{\infty} E_{\mathrm{t}}(\mathrm{n}) \mathrm{dn}=1
$$

The temperature spectral density is presented in a semi-logarithmic coordinate system, (in abscissa the $\mathrm{n}$ frequency and in ordinate the product $n \mathrm{E}_{\mathrm{t}}(\mathrm{n})$ ).

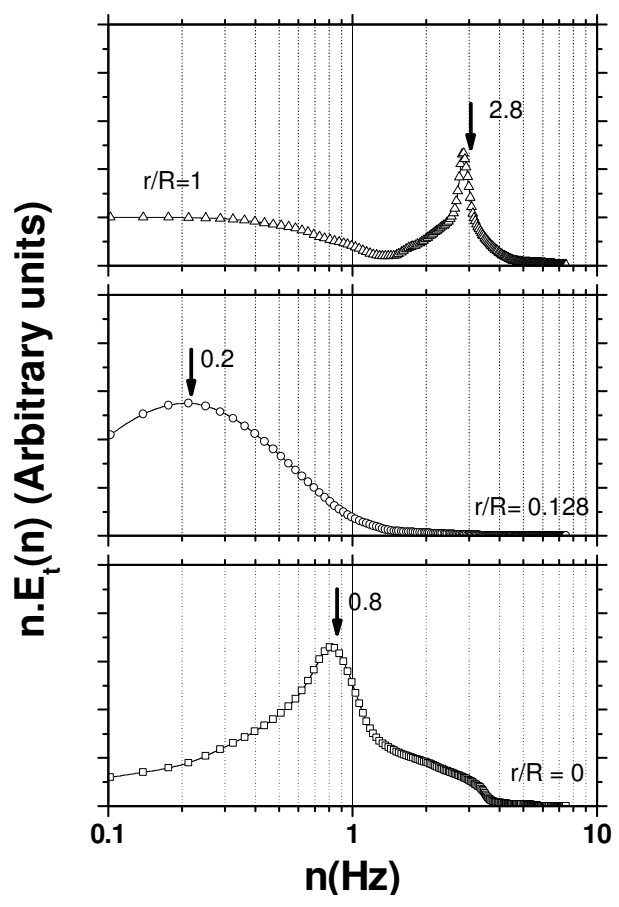

(a)

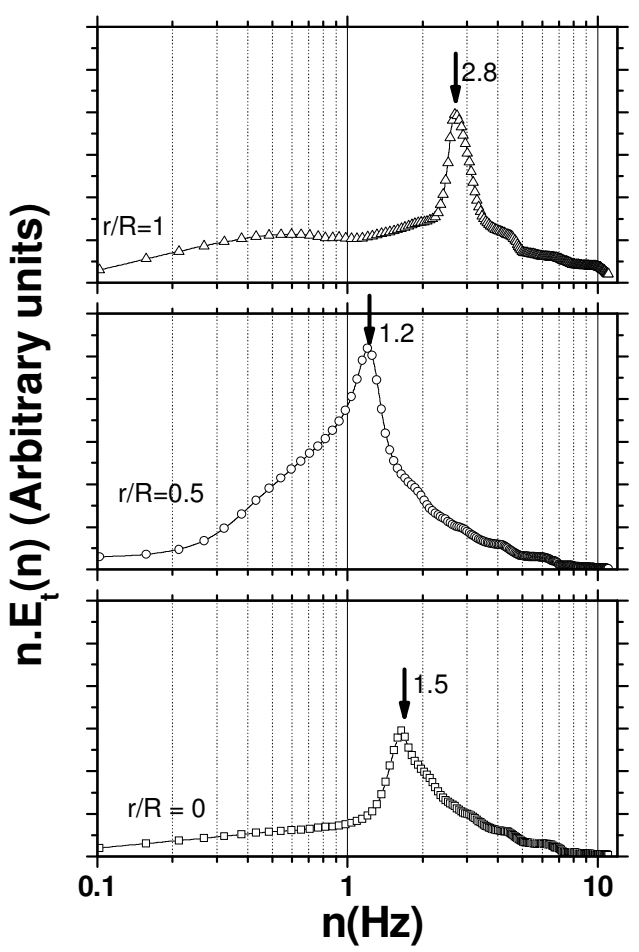

(b)

Fig. 9:Power spectral density of the temperature fluctuations: thermal plume evolving in an open environment -(a) $\mathrm{z} / \mathrm{D}=0.714$, (b) $\mathrm{z} / \mathrm{D}=4.28$

This representation permits the setting in evidence of the spectral strips containing a considerable energy, while taking in consideration the areas delimited by the spectra $^{[11,14,16]}$.

For the free plume, we present in the Fig. 9 temperature spectral density of the levels $(\mathrm{z} / \mathrm{D}=0.714$ and 4.28) and for three positions of the probe. 
For the level $\mathrm{z} / \mathrm{D}=0.714$, this figure shows that the spectral energy corresponding to the big structures (bearers of energy) is considerably more elevated than the one relative to the small dissipating structures. Indeed, at the source border $(\mathrm{r} / \mathrm{R}=1)$, the relative energy proportion to the structures on a big scale is contained in a narrow frequency strip understood between 0.1 and $1 \mathrm{~Hz}$. Thereafter, the spectral density presents a peak situated at $2.8 \mathrm{~Hz}$. This peak is bound to the fluid net coming from the low that comes to supply the hot surface of the source. In the region where the thermal turbulence intensity (It), is maximal $\left(\mathrm{r} / \mathrm{R}=0.128^{[10]}\right)$, one notes that the relative spectral energy to the structures on a big scale is situated in an included frequency strip between 0.1 and $1 \mathrm{~Hz}$. On the axis of the plume $(\mathrm{r} / \mathrm{R}=0)$, we notice that the spectral energy is concentrated around a frequency of $0.8 \mathrm{~Hz}$. The different spectral density shows that the major part of the energy is concentrated in a range of frequencies lower than $3 \mathrm{~Hz}$. It shows the predominance of the big vortex carriers of energy relatively in this zone close to the source of the plume.

In the zone where the turbulence is fully developed $(\mathrm{z} / \mathrm{D}=4.28)$, the peaks of spectral energy are located respectively at $2.8,1.2$ and $1.5 \mathrm{~Hz}$. we notice that the energy corresponding to the small dissipating structures increases whereas the one relative to the big structures decrease considerably.

For the plume with thermosiphon, Fig. 10 shows the spectral density at the levels $\mathrm{z} / \mathrm{D}=0.714$ and 4.28 for four positions of the probe. For the level $\mathrm{z} / \mathrm{D}=0.714$, the Fig. 10(a) shows some spectra in several peaks of energy that evolve according to the position of the probe. Indeed, very near of the cylinder wall $(\mathrm{r} / \mathrm{R}=2)$, the spectral density presents a peak at the frequency 1.1 Hz. As it has been shown by the visualization, this peak is bound to the fresh fluid thread close the wall. At the source border $(r / R=1)$, this figure shows a spectra in three peaks situated respectively at $1.3,2.3$ and $4.3 \mathrm{~Hz}$. These three peaks it's bound respectively to the three supply fluid threads what confirms the observations made during the visualization well. In the region where the thermal turbulence intensity is maximal $(\mathrm{r} / \mathrm{R}=0.5)$, this figure shows a peak at $1.7 \mathrm{~Hz}$. This peak characterizes the frequency of exhaust cycle of the rotating rolls. On the axis of the plume $(\mathrm{r} / \mathrm{R}=0)$, the spectral density shows the disappearance of the energy peaks, while leading to a strong decrease of energy from a frequency of the order of $0.1 \mathrm{~Hz}$. This behavior translates the character more laminar of the flow in the axis. On the other hand, the corresponding energy level to the big structures remains always very substantial in the level $\mathrm{z} / \mathrm{D}=0.714$ relatively close to the source.

For the high level ( $\mathrm{z} / \mathrm{D}=4.28)$, the Fig. 10(b) shows a shift of the energy peaks toward the high frequencies situated respectively at $4.4,5.3,2.7$ and $1.6 \mathrm{~Hz}$. It shows that the proportion of energy corresponding to the structures on a big scale decreases quickly, whereas

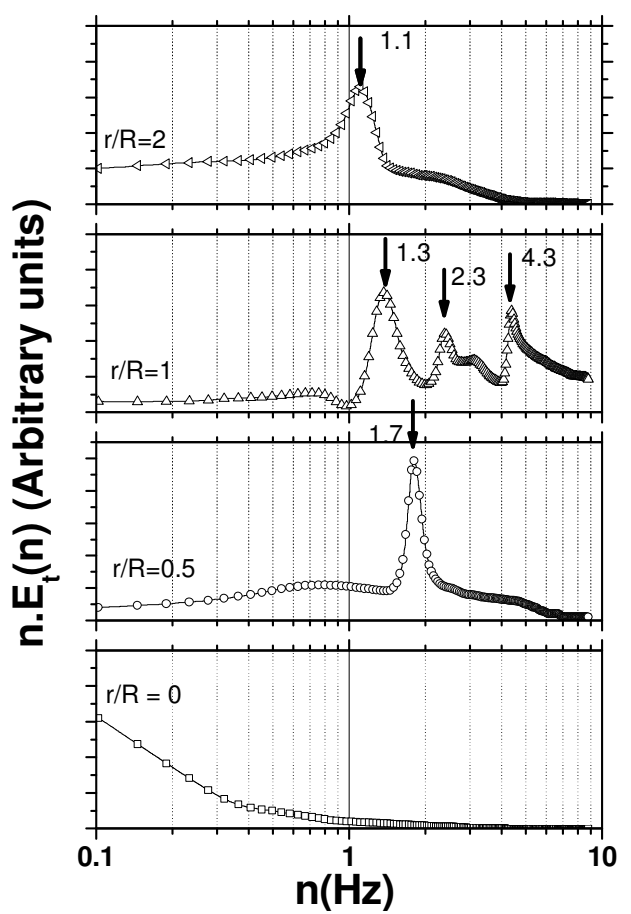

(a)

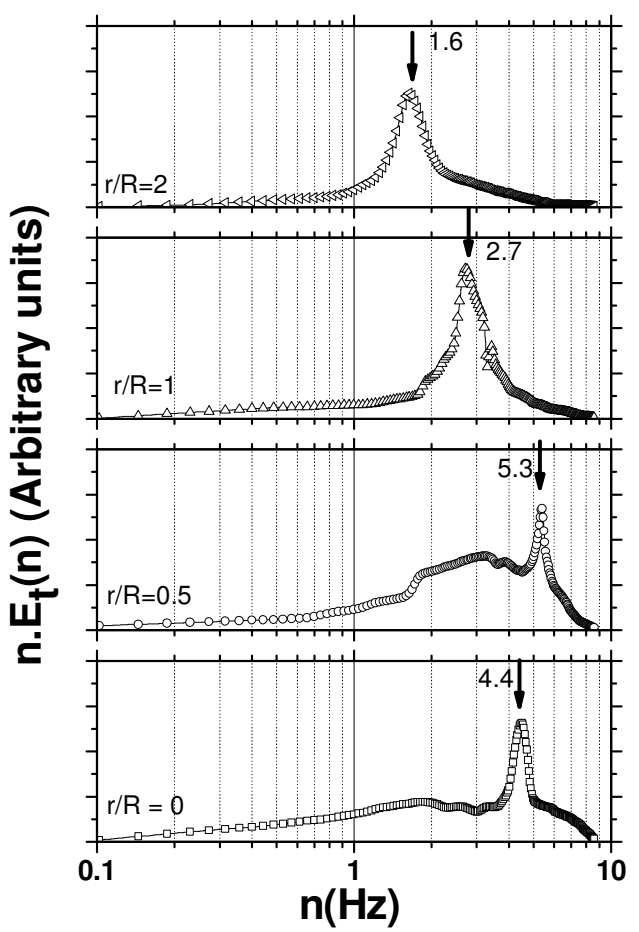

(b)

Fig. 10: Power spectral density of the temperature fluctuations: thermal plume evolving in an enclosed environment -(a) $\mathrm{z} / \mathrm{D}=0.714$, (b) $\mathrm{z} / \mathrm{D}=4.28$

the one relative to the small dissipating structures increases considerably.

We present in Fig. 11 a comparison of the energy spectra for the two studied cases and for the level $\mathrm{z} / \mathrm{D}=4$.28. Fig. 11 shows an important shift of the 


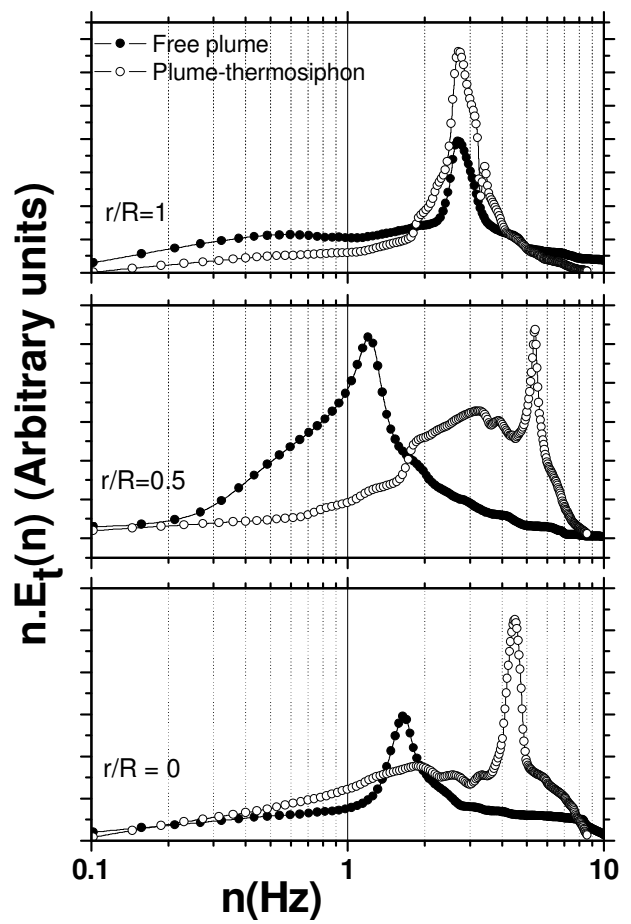

Fig. 11: Power spectral density of the temperature fluctuations: thermal plume evolving in an open environment and in an enclosed environment $-\mathrm{z} / \mathrm{D}=4.28$

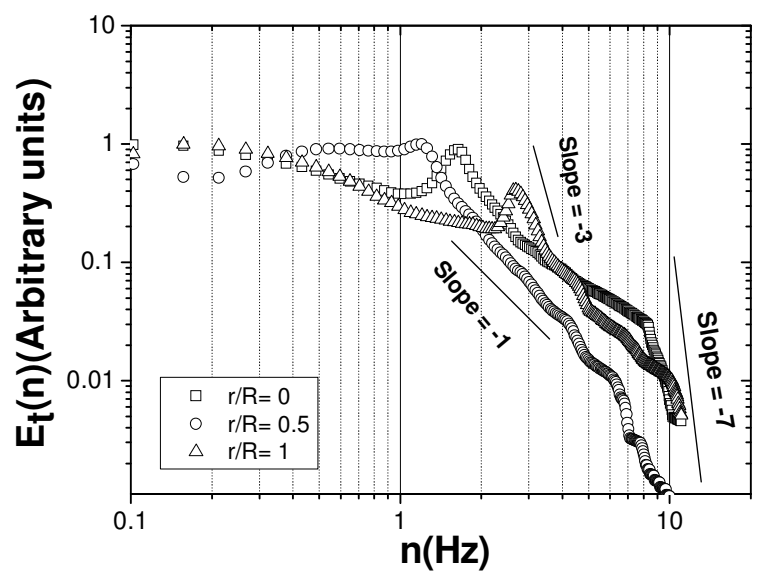

Fig. 12: Power spectral density of the temperature fluctuations: thermal plume evolving in an opened environment $-\mathrm{z} / \mathrm{D}=4.28$

energy peaks toward the high frequencies under the effect of the thermosiphon. As destroying structures them on a big scale generated by the plume, the thermosiphon provokes a fast mixture of the fluid thus while leading to vortex of weaker size.

Figures 12 and 13 show the plot of the normalized spectral energy as a function of the frequency (in LogLog coordinates). A typical spectrum of temperature fluctuations corresponding to an experimental configuration where the height measurement $\mathrm{z} / \mathrm{D}=4.28$. As the frequency increases, i.e. for smaller structures, the normalised energy tends to decrease. This behavior

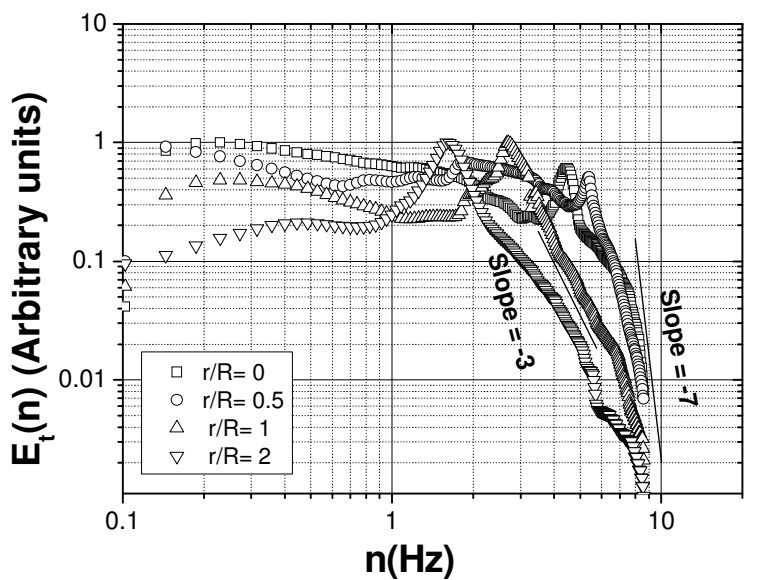

Fig. 13: Power spectral density of the temperature fluctuations: thermal plume evolving in an semi-enclosed environment - $\mathrm{z} / \mathrm{D}=4.28$

is consistent with the usual cascade scenario toward small length scales.

Three spectral regions can be identified in Fig. 12 and 13. The first region is a production region due to turbulent flux. The second region is quite interesting and is characterized by a law $\mathrm{n}^{-3}$, corresponding to a buoyancy region, typical in buoyant free convection flows. Such these behavior has been, already reported by Lumley ${ }^{[17]}$ in a spectral analysis of quasi-inertial turbulent flows in stably stratified environments and by several other authors for round plumes ${ }^{[4,5,18,19]}$. Rather than giving an analytical explanation of this -3 slope, we should emphasize the absence of the classical $-5 / 3$ slope, (although previous studies reported such behavior). We know that the existence of a constant $-5 / 3$ slope region is related to a hypothesis of homogeneity and isotropy. The appearance of a -3 power law region (inertial-diffusive sub range ${ }^{[4]}$ ) may be related with the passage in the measurement volume of large structures ${ }^{[18]}$ generated by the hot source. These structures are responsible for energy transfer modes that are not present in classical turbulent shear flows. Buoyancy effects seem to impose the rate at which potential energy is converted into kinetic energy of the larger scales. Taking over viscous effects (in comparison to flows for which energy transfers are driven essentially by turbulent shear), this additionally entails noticeable modifications of the inertial equilibrium range within which energy of the larger scales is transferred to smaller scales according to the cascade concept of Kolmogorov. We also observed the -1 slope (the Tchen law) for the free plume in the largefrequency region. The existence of the -1 slope region is related to an important turbulent production.

In the present study, a third region was also identified. This so-called dissipative region, of very fast decay, is consistent with an $\mathrm{n}^{-7}$ law. This kind of law was, proposed from theoretical considerations, by Corrsin ${ }^{[20]}$, in a study on temperature fluctuation spectra for isotropic turbulent flows. 


\section{CONCLUSION}

We have studied the turbulent fine structure of the thermal plume issued from a heated disk evolving in an unlimited and in a semi-enclosed environment. For the case of the unlimited environment, the visualization of the flow as well as the spectral study have show that the global structure of the flow is constituted of two very different zones; a first zone of the plume development, close to the source $(\mathrm{z} / \mathrm{D} \leq 2.14)$, and a second established turbulence zone relatively distant of the hot source ( $\mathrm{z} / \mathrm{D}>2.14)$. They also show the predominance of the structures on a big scale.

The experimental results have shown a considerable change of the guided flow structure (semienclosed environment) in comparison with the case of the free flow. Indeed, for the guided flow case, the visualization shows in the source vicinity, the appearance of a supplementary zone that is added at the two classic zones, characterize the vertical evolution of the plume. This zone is characterized by the formation in the hot source surface the two symmetrical rotating rolls.

For the case of semi-enclosed environment, this study shows that the structure of the resulting flow is divides in three zones: A first zone of instability close to the source ( $\mathrm{z} / \mathrm{D} \leq 0.714)$, where the strong plumethermosiphon interaction entailed the apparition of the rotating rolls. A second intermediate zone $(0.714<\mathrm{z} / \mathrm{D}$ $\leq 2.14$ ), where develops the interaction flow. A last zone $(\mathrm{z} / \mathrm{D}>2.14)$, where the turbulence is fully developed, that occupies the superior part of the cylinder.

This study also shows that the energy proportion corresponding to the small structures (dissipating) in the semi-enclosed environment is more important than in the bigger structures encountered for the unlimited environment. The formation of these turbulent dissipating structures leads to a uniformity flow in the upper part of the cylinder.

The spectra for temperature fluctuations obtained enabled identifying three distinct spectral regions that correspond to energy transfer mechanisms between scales: a production region due to turbulent flux; a buoyancy region characterized by a $\mathrm{n}^{-3}$ law, characteristic of anisotropic turbulence caused by buoyancy that modifies the inertial equilibrium range within which energy of the larger scales is transferred to smaller scales and finally, a dissipation region that follows a behavior as per $\mathrm{n}^{-7}$. The $\mathrm{n}^{-1}$ Tchen law appears clearly for the free plume that related to an important turbulent production.

\section{Nomenclature}

$\mathrm{D}=$ diameter of the disk heated source, $\mathrm{m}$

$r=$ radial coordinate, $m$

$R=$ radius of the disk heated source, $\mathrm{m}$

$z=$ vertical coordinate, $m$

\section{REFERENCES}

1. George, W.K., R.L. Alpert and F. Tamanini, 1977. Turbulence measurements in an axisymmetric buoyant plume. Intl. J. Heat Mass Transfer, 20: 1145-1154.

2. Shabbir, A. and W.K. George, 1994. Experiments in a round turbulent buoyant plume. J. Fluid Mech., 275: 1-32.

3. So, R.M.C. and H. Aksoy, 1993. On vertical turbulent buoyant jets. Intl. J. Heat Mass Transfer, 36: 3187-3200.

4. Dai, Z., L.K. Tseng and G.M. Faeth, 1994. Structure of round, fully developed, buoyant turbulent plumes. J. Heat Transfer, 116: 409-417.

5. Dai, Z., L.K. Tseng and G.M. Faeth, 1995. Velocity statistics of round, fully developed, buoyant turbulent plumes. J. Heat Transfer, 117: 138-145.

6. Brahimi, M. and D. Kim-Son, 1986. Experimental and numerical predictions of the mean flow of a turbulent pure plume. Arch. Mech. Warszawa, 38: 519-528.

7. Mahmoud, A.O.M., R. B. Maad and A. Belghith, 1998. Interaction d'un écoulement de thermosiphon avec un panache thermique à symétrie axiale: étude expérimentale. Rev. Gén. Therm., 37: 385-396.

8. Mahmoud, A.O.M., R.B. Maad and A. Belghith, 1998. Production of hot air with quasi-uniform temperature using concentrated solar radiation. Renewable Energy, 13: 481-493.

9. Zinoubi, J., 2003. Etude de l'interaction d'un écoulement de thermosiphon avec un panache thermique à symétrie axiale:Influence de paramètre de forme. Thèse de Doctorat, Université Elmanar, Faculté des Sciences de Tunis.

10. Zinoubi, J., R.B. Maad and A. Belghith, 2004. Influence of the vertical source-cylinder spacing on the interaction of thermal plume with a thermosiphon flow: An experimental study. Exp Therm. Fluid. Sci., 28 : 329-336.

11. Agator, J.M., 1983. Contribution à l'étude de la structure turbulente d'un panache thermique à symétrie axiale. Interaction du panache avec son environnement limité, Thèse, Université de Poitiers.

12. Pera, L. And B. Gebhart, 1975. Laminar plume interactions. J. Fluid Mech., 68: 259-271.

13. Brahimi, M., L. Dehmani and D. Kim-Son., 1989. Structure turbulente de l'écoulement d'interaction de deux panaches thermiques. Intl. J. Heat Mass Transfer, 32: 1551-1559.

14. Brahimi, M., 1987. Structure turbulente des panaches thermiques-interaction. Thèse, Université de Poitiers. 
15. Brahimi, M., M. Lamour and D. Kim-Son, 1988. Champs moyens et fluctuants des panaches thermiques isolés ou en interaction. Rev. Gen. Therm., 315-316: 236-243.

16. Kim-Son, D., 1977. Contribution à l'étude de la zone de transition et de la zone de turbulence établie dans un écoulement de convection naturelle sur une plaque plane verticale isotherme. Thèse de Doctorat d'Etat, Université de Poitiers.

17. Lumley, J.L., 1965. The spectrum of nearly inertial turbulence in a stably stratified fluid. J. Atmos. Sci., 21: 99-102.
18. Kotsovinos, N., 1991. Turbulence spectra in free convection flow. Phys. Fluids A, 3: 163-167.

19. Sangras, R., Z. Dai and G.M. Faeth, 1988. Mixing structure of plane self-preserving buoyant turbulent plumes. ASME J. Heat Transfer, 120: 1033-1041.

20. Corrsin, S., 1951. On the spectrum of isotropic temperature fluctuations in an isotropic turbulence. J. Appl. Phys., 22: 469-473. 